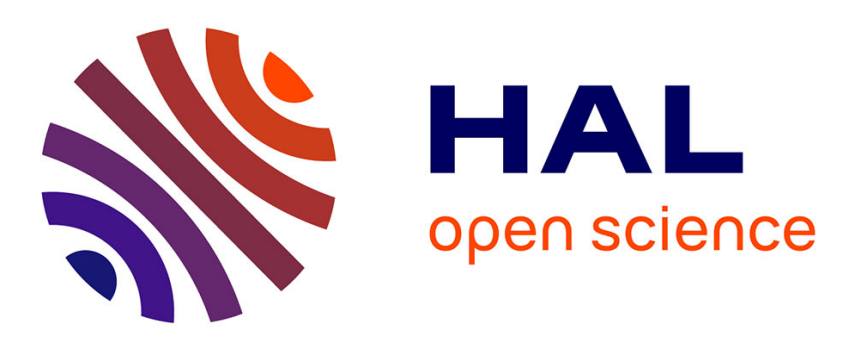

\title{
Recherche de corrélations simples exprimant les pertes convectives dans une cavité bidimensionnelle, inclinée, chauffée différentiellement
}

\author{
J.C. Grondin, B. Roux
}

\section{- To cite this version:}

J.C. Grondin, B. Roux. Recherche de corrélations simples exprimant les pertes convectives dans une cavité bidimensionnelle, inclinée, chauffée différentiellement. Revue de Physique Appliquée, 1979, 14 (1), pp.49-56. 10.1051/rphysap:0197900140104900 . jpa-00244590

\author{
HAL Id: jpa-00244590 \\ https://hal.science/jpa-00244590
}

Submitted on 1 Jan 1979

HAL is a multi-disciplinary open access archive for the deposit and dissemination of scientific research documents, whether they are published or not. The documents may come from teaching and research institutions in France or abroad, or from public or private research centers.
L'archive ouverte pluridisciplinaire HAL, est destinée au dépôt et à la diffusion de documents scientifiques de niveau recherche, publiés ou non, émanant des établissements d'enseignement et de recherche français ou étrangers, des laboratoires publics ou privés. 


\title{
Recherche de corrélations simples exprimant les pertes convectives dans une cavité bidimensionnelle, inclinée, chauffée différentiellement $\left(^{*}\right)$
}

\author{
J. C. Grondin et B. Roux \\ Institut de Mécanique des Fluides \\ 1, rue Honnorat, 13003 Marseille, France \\ (Reçu le 28 juin 1978, révisé le 11 septembre 1978, accepté le 14 septembre 1978)
}

\begin{abstract}
Résumé. - On cherche à corréler les résultats numériques du flux de chaleur obtenus en résolvant, par une méthode aux différences finies de haute précision, les équations qui décrivent le mouvement de convection naturelle laminaire dans une cavité inclinée, différentiellement chauffée. Après une étude critique des corrélations déjà proposées dans la littérature, la loi simple suivante $: \overline{N u}=A R a^{a} l^{-b}$ exprimant le nombre de Nusselt en fonction des principaux paramètres du problème, a été retenue. L'expression des coefficients $A, a$ et $b$, pour différents angles d'inclinaison $\alpha_{c}<\alpha<120^{\circ}$ où l'écoulement est bidimensionnel, a été proposée dans le cas du régime de couche limite.
\end{abstract}

Abstract. - We tried to correlate the computed values of the heat transfer obtained in solving, by a high order accurate finite difference method, the equations describing the natural convection in an inclined cavity, differentially heated. After a review of the correlations previously reported in the literature, the following expression : $\overline{N u}=A R a^{a} l^{-b}$, giving the Nusselt number in terms of the main parameters of the problem, has been adopted. The values of coefficients $A, a$ and $b$, for different inclination angles $\alpha_{c}<\alpha<120^{\circ}$ for which the flow is twodimensional, has been proposed for the boundary layer regime.

\section{Nomenclature}

$C_{\mathrm{p}}$

$g$

Gr

$H$

$k$

$L$

$l$

M

$m$

$\frac{N u}{N u}$

$\overline{N u}$

$p$

$\operatorname{Pr}$

$R a$

nombre de Nusselt local $\overline{N u}=\frac{1}{l} \int_{0}^{l} N u \mathrm{~d} x$ $\operatorname{Pr}=C_{\mathrm{p}} \mu / k=v / \chi$

Nomenclature
chaleur spécifique à pression constante du
fluide

accélération de la pesanteur

nombre de Grashof défini par $G r=R a / P r$

hauteur de la cavité contenant le fluide conductivite thermique du fluide

longueur de la cavité contenant le fluide

facteur de forme de la cavité $l=L / H$

largeur de la cavité contenant le fluide

facteur de forme de la cavité $m=M / H$

nombre de Nusselt moyen défini par

pression au sein du fluide

nombre de Prandtl du fluide défini par

nombre de Rayleigh défini par

$R a=\left(\frac{g \beta \rho C_{\mathrm{p}}}{\mu k}\right) \Delta T H^{3}=\left(\frac{g \beta}{v \chi}\right) \Delta T H^{3}$

(*) Cette étude a été réalisée dans le cadre de l'Action Thématique Programmée Habitat solaire du C.N.R.S. avec la collaboration du Département d'Héliophysique de l'Université de Provence.

$t$
$T$
$T_{\mathrm{p}}$
$T_{\mathrm{v}}$
$T_{0}$
$u$
$U$

angle d'inclinaison de la couche fluide par rapport à l'horizontale

$\beta \quad$ coefficient volumique d'expansion thermique du fluide

rotationnel de vitesse défini par $\zeta=-\nabla^{2} \psi$

diffusivité thermique du fluide $\chi=k / \rho C_{\mathrm{p}}$

température adimensionnelle $\theta=\frac{T-T_{\mathrm{a}}}{\Delta T}$ 
$\mu \quad$ viscosité absolue du fluide

$v \quad$ viscosité cinématique du fluide $v=\mu / \rho$

$\rho \quad$ masse volumique du fluide

$\tau \quad$ temps adimensionnel $\tau=\frac{t \chi}{H^{2}}$

$\psi$

fonction de courant définie par $U=\partial \psi-\partial Y$ et $V=-\partial \psi / \partial X$.

Opérateurs :

$\nabla^{2}=\frac{\partial^{2}}{\partial X^{2}}+\frac{\partial^{2}}{\partial Y^{2}}$

$\frac{D}{D t}=\frac{\partial}{\partial t}+u \frac{\partial}{\partial x}+v \frac{\partial}{\partial y}$

$\frac{D}{D \tau}=\frac{\partial}{\partial \tau}+U \frac{\partial}{\partial X}+V \frac{\partial}{\partial Y}$.

1. Introduction. - Le bilan thermique d'un convertisseur de l'énergie solaire dépend de deux processus de pertes de chaleur qui sont :

- les pertes par rayonnement,

- les pertes par convection et conduction.

Pour l'essentiel, ces pertes ont lieu par la face avant du dispositif, c'est-à-dire celle qui reçoit le rayonnement solaire incident. La connaissance de ces deux phénomènes est donc nécessaire pour établir, prévoir, ou améliorer le bilan thermique; le convertisseur idéal étant celui pour lequel les pertes sont nulles.

Un cas particulier de convertisseur thermique de l'énergie solaire, présentant un intérêt important au point de vue de ses applications, est celui du capteur plan avec effet de serre. Dans ce cas, la géométrie du dispositif est simple puisqu'il est constitué de deux surfaces planes, en regard l'une de l'autre, l'absorbeur et la couverture de verre, tandis que les parois latérales sont faites d'un matériau isolant. De tels capteurs sont déjà construits par l'industrie pour chauffer un fluide caloporteur à des températures de l'ordre de $50^{\circ} \mathrm{C}$. Actuellement on cherche à élever cette température en employant des absorbeurs sélectifs destinés à réduire les pertes par rayonnement infrarouge. Si l'on admet que l'on puisse réduire ces pertes de façon notable, les pertes par convection prennent alors une grande importance. Pour la gamme des capteurs fonctionnant à moyenne température il est donc nécessaire de connaître le flux de chaleur perdu par convection naturelle et ceci en faisant abstraction, dans un premier temps, de la convection forcée due au vent et d'une interaction convection-rayonnement.

Le présent article rassemble les principaux résultats d'une étude numérique qui est développée en détails dans le mémoire de thèse d'un des auteurs [1].

Cette étude a été réalisée dans le cadre de certaines hypothèses simplificatrices. En particulier, on a négligé l'influence du circuit de récupération qui induit, naturellement, un gradient de température le long de l'absorbeur, puisque le fluide caloporteur subit un accroissement de température entre l'entrée et la sortie. L'absorbeur et la vitre sont donc considérés avec une température de surface homogène. Par ailleurs le mouvement de l'air dans l'espace couvertureabsorbeur est considéré comme stationnaire, laminaire et bidimensionnel (Fig. 1).

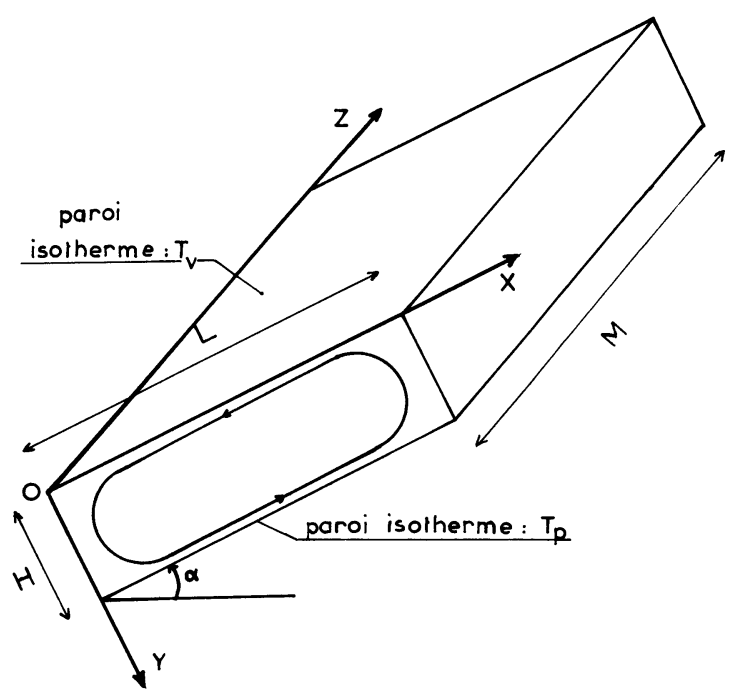

Fig. 1. - Système des coordonnées.

[Coordinate system.]

2. Discussion des hypothèses de base. - Contrairement au cas d'une couche fluide horizontale chauffée par en dessous, il existe dans une couche fluide inclinée un mouvement de base dès qu'apparaît le plus léger gradient de température au sein de ce fluide. Cet écoulement de base, unicellulaire et bidimensionnel, est caractérisé par un mouvement ascendant le long de la plaque chaude et descendant sur la plaque froide.

Deux types de perturbations stationnaires peuvent se superposer à ce mouvement de base suivant les valeurs de l'angle d'inclinaison $\alpha$; comme l'ont montré notamment Hart [2], Korpela [3] et Birikh et al. [4], il s'agit :

1) de perturbations longitudinales (Fig. 2) pour $0<\alpha<\alpha_{c}$. Elles se présentent sous forme de rouleaux contrarotatifs d'axes orientés suivant $O x$, et se superposent au mouvement de base pour donner un écoulement d'ensemble tridimensionnel dont il ne sera pas question ici ;

2) de perturbations transverses (Fig. 3) pour $\alpha_{c}<\alpha<180^{\circ}$. Elles se manifestent par des rouleaux co-rotatifs d'axes orientés suivant $O z$ qui ne détruisent donc pas le caractère bidimensionnel du mouvement de base.

La valeur $\alpha_{c}$ de l'angle de transition entre ces deux types d'instabilité est une variable complexe de 
$\mathrm{Ra}=3500 \quad \ell=10$

Lignes de courant

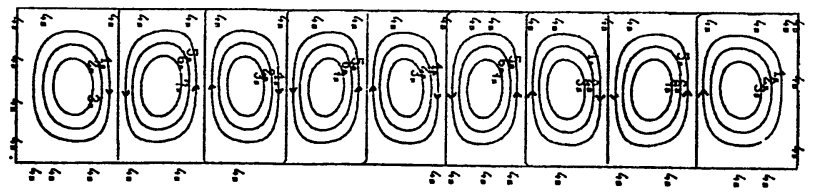

Isothermes

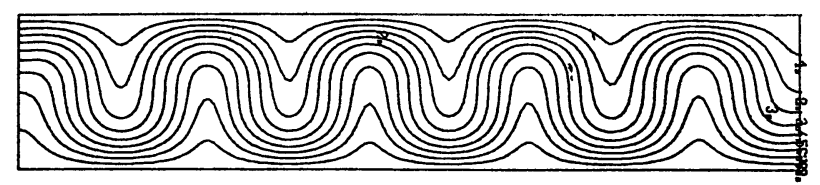

Fig. 2. - Lignes de courant et isothermes dans le cas horizontal.

[Streamlines and isotherms in the horizontal case.]

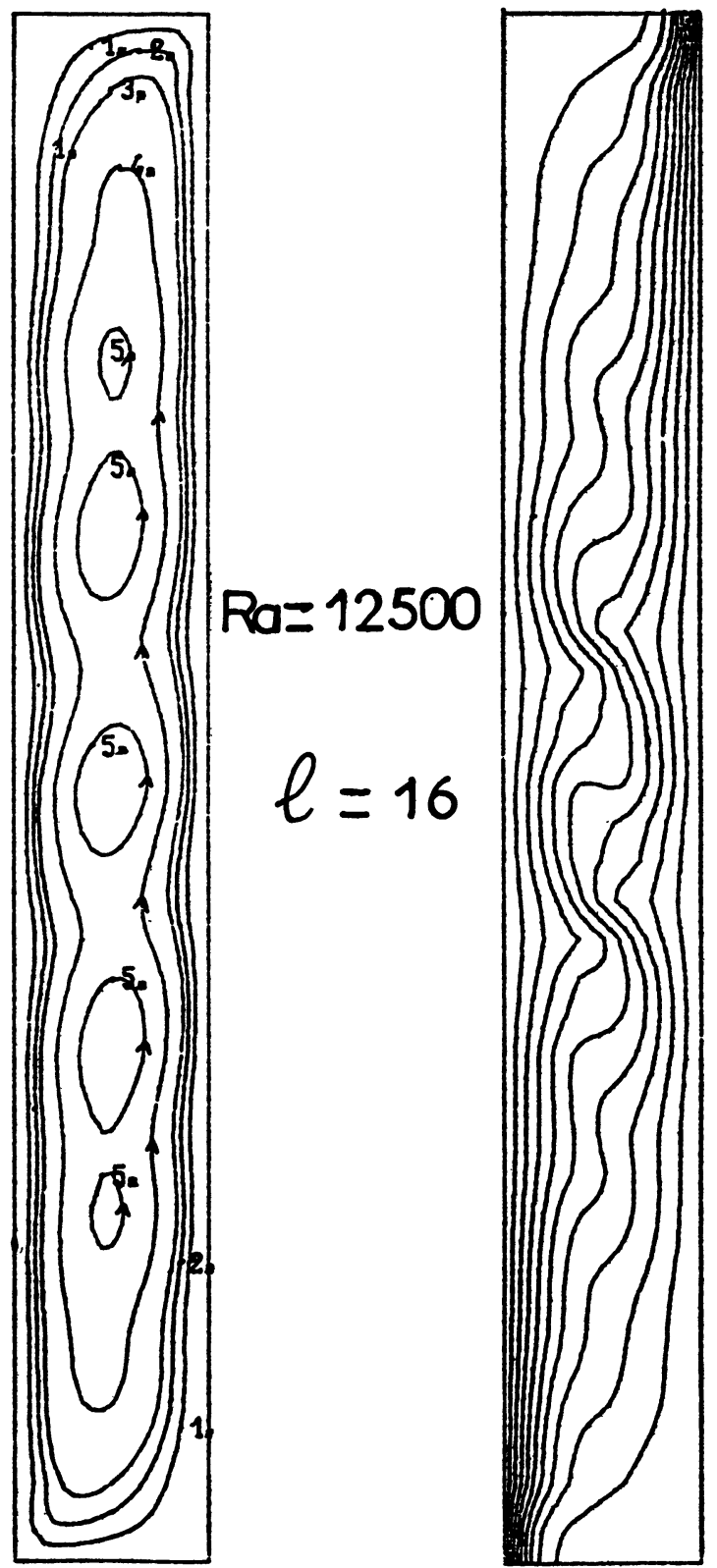

Fig. 3. - Lignes de courant et isothermes dans le cas vertical.

[Streamlines and isotherms in the vertical case.] la nature du fluide et des facteur de forme $l$ et $m$ de la cavité à l'intérieur de laquelle sont observées ces perturbations.

On peut néanmoins distinguer deux cas très simples. D'une part si la couche fluide est d'extension très grande ( $l$ et $m$ infinis), les études de Hart [2], Korpela [3], Birikh et al. [4], Unny [5] et Chandrasekhar [6] ont montré que cet angle serait de l'ordre de $70^{\circ}$ pour l'air $(\operatorname{Pr}=0,7)$ et peu différent de $90^{\circ}$ pour des fluides ayant un haut nombre de Prandtl (Figures 1-3 de la référence [1]). D'autre part si $l$ est de l'ordre de l'unité, Ozoe et al. [7] ainsi que Arnold et al. [8] n'ont pu mettre en évidence l'existence d'instabilités longitudinales que pour des angles très faibles, et celà pour des écoulements d'huile au silicone $(\operatorname{Pr}>1000)$ ou d'eau $(\operatorname{Pr}=4,5)$. La valeur de $\alpha_{c}$ peut donc varier de quelques degrés à quatre-vingtdix degrés suivant les cas considérés.

Par ailleurs, lorsque le nombre de Rayleigh devient assez grand, il peut exister dans la couche fluide des perturbations instationnaires. Celles-ci pouvant se manifester, soit sous la forme de rouleaux de perturbations transverses instationnaires, soit sous forme de phénomènes transitoires analogues au phénomène de convection fluctuante observé dans une couche poreuse par Bories et Combarnous [9]. On peut trouver dans la littérature quelques valeurs du nombre de Rayleigh de transition entre un écoulement laminaire stationnaire et un écoulement instationnaire. Pour une couche d'air horizontale chauffée par en dessous, la valeur $R a_{t} \simeq 7 \times 10^{4}$ a été proposée par Giblin [10]. Pour une couche verticale, une étude de Mordchelles-Regnier et Kaplan [11] nous montre que $R a_{t}$ dépend de l'allongement $l$ et se situe ainsi aux alentours de $2 \times 10^{5}$ pour des allongements assez importants $(l \simeq 25)$.

Devant les difficultés de donner une valeur unique à $\alpha_{\mathrm{c}}$ et à $R a_{t}$ des calculs ont été faits dans la gamme : $0<\alpha<180^{\circ}$ et $0<R a<2 \times 10^{5}$.

Mais ils ne sont strictement valables que pour : $\alpha_{c}<\alpha<180^{\circ}$ et $0<R a<R a_{t}$.

3. Equations de la convection. - Les équations sont écrites pour un écoulement laminaire et bidimensionnel en supposant vraies les hypothèses suivantes :

1) La masse volumique $\rho$ est constante sauf dans l'expression de la force d'Archimède (hypothèse de Boussinesq). Cette masse volumique s'exprime alors comme :

$\rho=\rho_{0}\left(1-\beta\left(T-T_{0}\right)\right)$

où $\rho_{0}$ désigne la masse volumique à la température moyenne $T_{0}$ et $\beta$ le coefficient d'expansion thermique du fluide.

2) Les propriétés de transport décrites à l'aide des paramètres $v$ et $\chi$ (viscosité et diffusivité thermique du fluide) sont supposées constantes et calculées à la température moyenne $T_{0}$. 
3) La dissipation visqueuse est négligeable dans l'équation de l'énergie.

Ces hypothèses ne sont valables en toute rigueur que lorsque les différences de température $\Delta T$ sont faibles. Gray et Giorgini [12] ont récemment donné un ordre de grandeur des différences de température pour lequel elles sont applicables dans le cas d'une couche d'air. Il semble pourtant que la valeur $\Delta T=28,6^{\circ} \mathrm{C}$ de ces auteurs soit assez restrictive.

3.1 EQUATIONS DE LA CONVECTION NATURELLE. Si $u$ désigne la composante de la vitesse suivant la direction $O x, v$ la composante suivant $O y, p$ la pression, $g$ l'accélération de la pesanteur, $T$ la température et $\alpha$ l'angle d'inclinaison de la couche fluide, ces équations s'écrivent :

a) équation de continuité

$\frac{\partial u}{\partial x}+\frac{\partial v}{\partial y}=0$

b) équations du mouvement

$\frac{D u}{D t}=-\frac{1}{\rho_{0}} \frac{\partial p}{\partial x}+v \nabla^{2} u-\frac{\rho}{\rho_{0}} g \sin \alpha$
$\frac{D v}{D t}=-\frac{1}{\rho_{0}} \frac{\partial p}{\partial y}+v \nabla^{2} v+\frac{\rho}{\rho_{0}} g \cos \alpha ;$

c) équation de conservation de l'énergie

$\frac{D T}{D t}=\chi \nabla^{2} T ;$

d) conditions aux limites : les conditions d'adhérence à la paroi s'expriment par :

$u(t, 0, y)=u(t, L, y)=u(t, x, 0)=u(t, x, H)=0$

$v(t, 0, y)=v(t, L, y)=v(t, x, 0)=v(t, x, H)=0$

$\forall x \in(0, L), \forall y \in(0, H)$.

Tandis que le caractère isotherme de la vitre et de l'absorbeur, ainsi que le caractère adiabatique des parois latérales s'écrivent :

$T(t, x, 0)=T_{v} ; \quad T(t, x, H)=T_{\mathrm{p}}, \quad \forall x \in(0, L)$

$\frac{\partial T}{\partial x}(t, 0, y)=\frac{\partial T}{\partial x}(t, L, y)=0, \quad \forall y \in(0, H)$.

3.2 Forme ADIMENSIONNELlE. - Ces équations sont développées dans leur forme instationnaire en utilisant la fonction de courant $\Psi$, le rotationnel de la vitesse $\zeta$ et la température $\theta$ comme variables dépendantes; $\Psi, \zeta$ et $\theta$ sont définis tels que :

$$
\begin{array}{ll}
U=\frac{H}{x} u=\frac{\partial \Psi}{\partial Y}, & V=\frac{H}{x} v=-\frac{\partial \Psi}{\partial X} \\
\zeta=-\nabla^{2} \Psi, & \theta=\frac{T-T_{0}}{T_{\mathrm{p}}-T_{\mathrm{v}}}=\frac{T-T_{0}}{\Delta T} .
\end{array}
$$

La pression est éliminée par une différentiation et une combinaison adéquate des équations du mouvement. Enfin l'équation de continuité elle-même est rendue pseudo-instationnaire et nous nous intéressons à la limite stationnaire quand le temps $\tau$ tend vers l'infini. On a alors un système d'équations aux dérivées partielles se présentant sous la forme donnée par Ozoe [13] :

$$
\begin{aligned}
& \frac{1}{P r} \frac{D \zeta}{D \tau}=-R a\left(\frac{\partial \theta}{\partial Y} \sin \alpha+\frac{\partial \theta}{\partial X} \cos \alpha\right)+\nabla^{2} \zeta \\
& \frac{D \theta}{D \tau}=\nabla^{2} \theta \\
& -\nabla^{2} \Psi+\frac{\partial \Psi}{\partial \tau}-\zeta=0
\end{aligned}
$$

avec les conditions aux limites suivantes :

$\theta(X, 0, \tau)=-\frac{1}{2} ; \quad \theta(X, 1, \tau)=+\frac{1}{2}$

$\frac{\partial \theta}{\partial X}(0, Y, \tau)=\frac{\partial \theta}{\partial X}(l, Y, \tau)=0$

$\forall X \in(0, l), \quad \forall Y \in(0,1)$

$\Psi=\frac{\partial \Psi}{\partial \mathbf{n}}=0$ sur le contour

(n désignant la normale à la paroi).

Il est intéressant de remarquer qu'il n'existe aucune condition aux limites explicite sur le rotationnel de la vitesse, tandis que les conditions aux limites sur la fonction de courant sont en surnombre. Il s'agit alors d'exprimer $\zeta$, sur la paroi, en fonction de $\Psi$. Sur un bord solide, la condition devient :

$\zeta_{\text {bord }}=-\left(\frac{\partial^{2} \Psi}{\partial \mathbf{n}^{2}}\right)_{\text {bord }}$.

Les conditions (3.6) seront utilisées dans l'expression discrétisée de (3.7).

Chacune de ces équations aux dérivées partielles est ramenée à une forme différentielle ordinaire par un schéma implicite aux directions alternées (A.D.I.).

Cette forme différentielle ordinaire est elle-même traitée par une méthode aux différences finies efficace et de haute précision dont le principe est donné en référence [14]. Les détails de l'application de cette méthode au cas des équations (3.1) à (3.7) sont donnés en références [1] et [15].

4. Régimes d'écoulement. - Différents régimes ont été identifiés dans le cas particulier où $\alpha=90^{\circ}$ par Eckert et Carlson [15], puis par Thomas et De Vahl Davis dans [16].

On peut ainsi voir (Fig. 4) qu'un mouvement de base existe déjà pour $R a=1600$, lorsque $l=8$. Néanmoins pour une telle valeur de $R a$ ce mouvement n'affecte guère que les extrémités de la cavité ; 
conductif
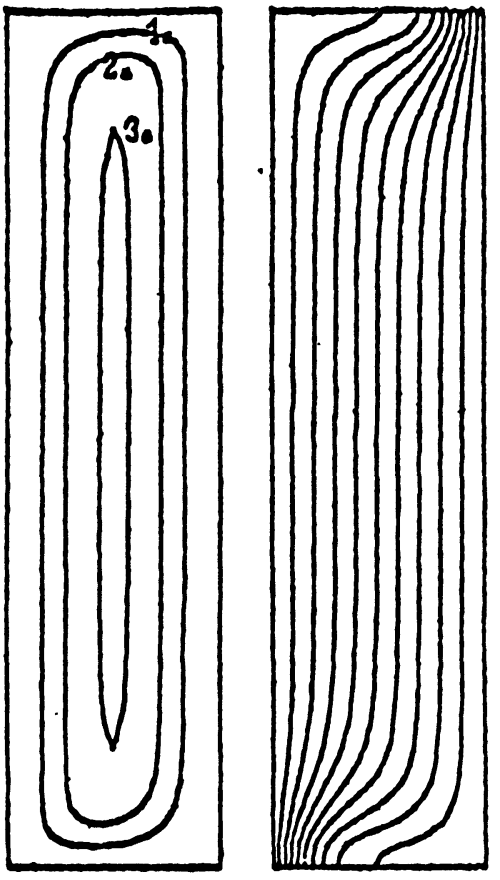

$R a=1600$ transitoire
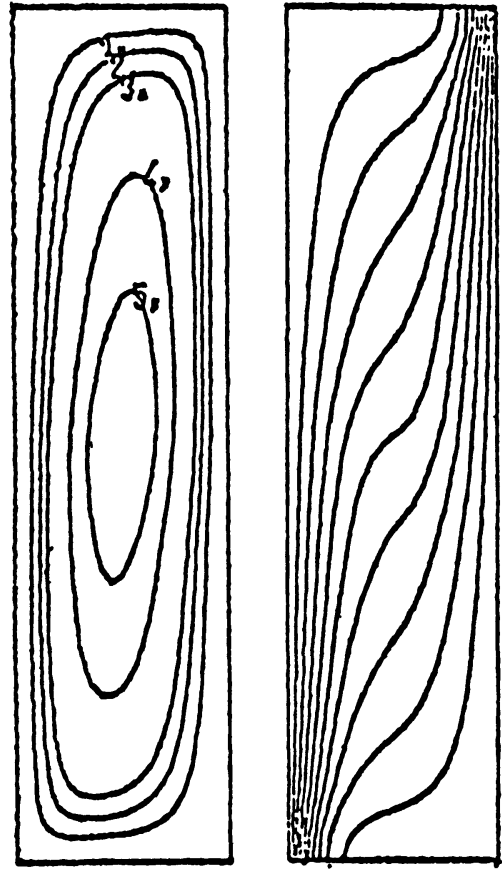

$R a=12500$ couche limite
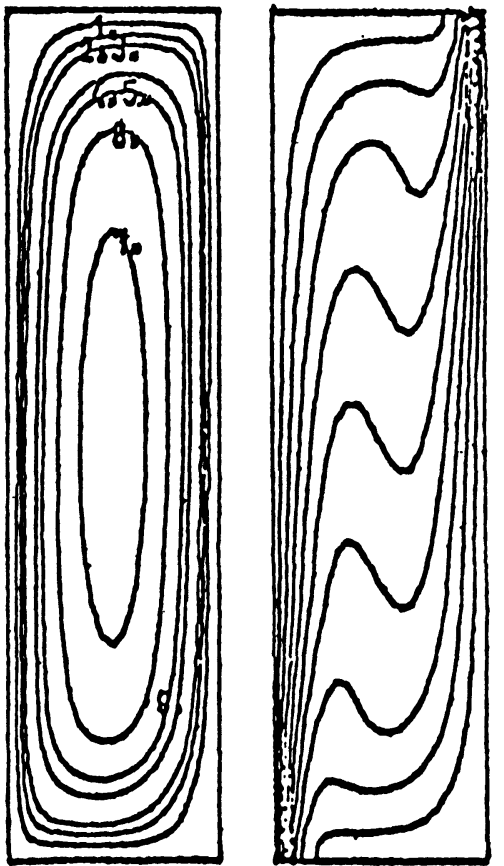

$R a=100000$

Fig. 4. - Régimes d'écoulement dans le cas vertical $(l=8)$.

[Regimes flow in the vertical case $(l=8)$.]

dans la majeure partie de celle-ci le transfert thermique s'effectue par conduction et les isothermes restent parallèles aux parois. Ce régime est communément appelé régime de type conductif. La valeur du gradient horizontal de température du point

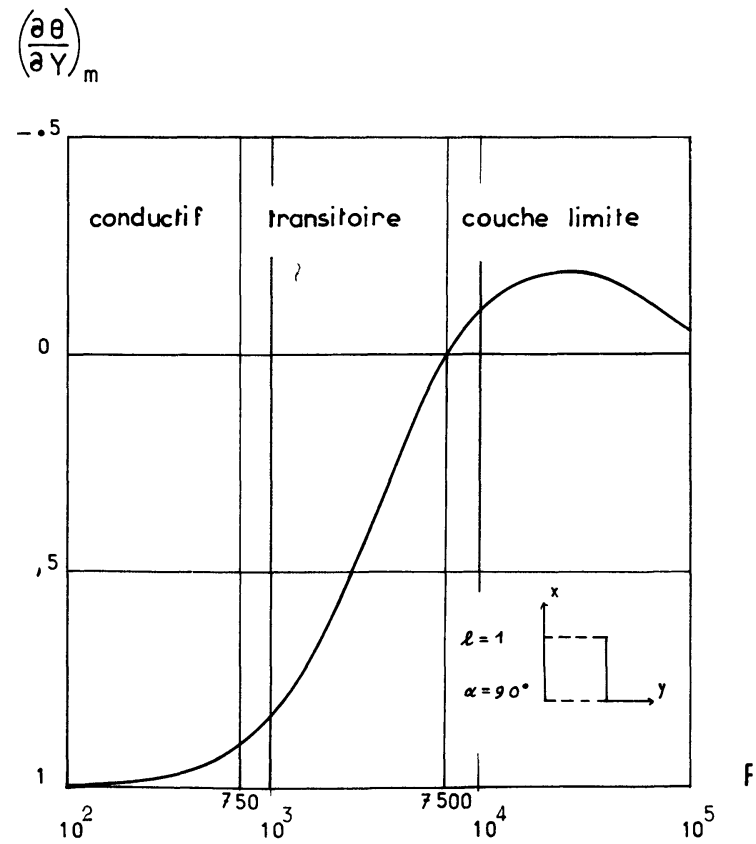

Fig. 5. - Evolution du gradient horizontal de température $(l=1)$. [Evolution of the horizontal temperature gradient $(l=1)$.] milieu de la cavité $(\partial \theta / \partial y)_{m}$, reste près de la valeur correspondant au régime de conduction pure. $\mathrm{Au}$ contraire, dans le régime de couche limite, $(\partial \theta / \partial y)_{m}$ tend vers 0 avec l'augmentation de $R a$ (Figs. 5 et 6 ).

On caractérisera, comme en référence [16], la fin

$\left(\frac{\partial \theta}{\partial Y}\right)_{m}$

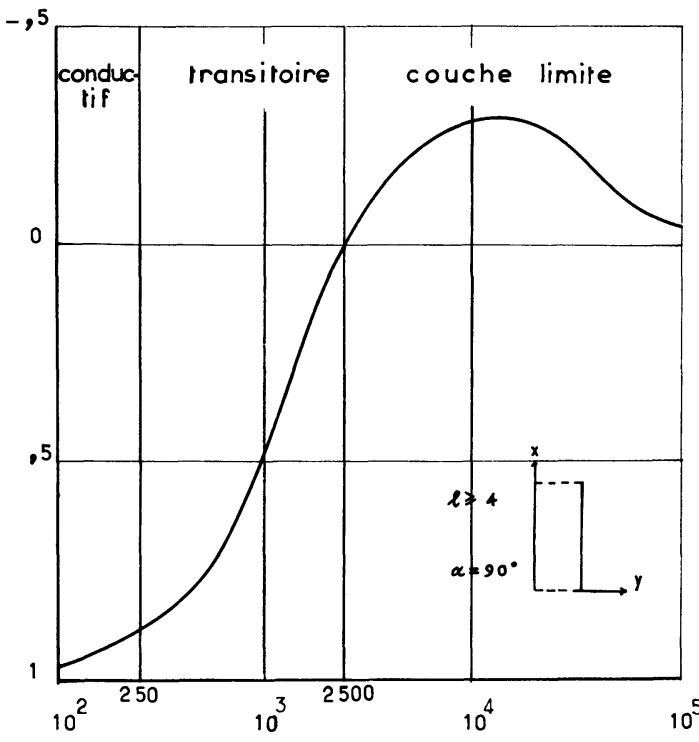

$\mathrm{Ra}$

Fig. 6. - Evolution du gradient horizontal de température $(l>4)$.

[Evolution of the horizontal temperature gradient $(l>4)$.] 
du régime conductif pour $R a=R a_{\mathrm{cd}}$ tel que $(\partial \theta / \partial y)_{m}=0,9$ et le début du régime de couche limite pour $R a=R a_{\mathrm{cl}}$ tel que $(\partial \theta / \partial y)_{m}=0$. On obtient ainsi, pour $l=1, R a_{\mathrm{cd}}=750$ et $R a_{\mathrm{c} l}=7500$ (Fig. 5). Dans le cas $l \geqslant 4$, on a $R a_{\mathrm{c} d}=250 l$ et $R a_{\mathrm{c} l}=2500 l$.

5. Transfert de chaleur. - Le transfert de chaleur local peut être caractérisé par le nombre de Nusselt $\mathrm{Nu}$, rapport du transfert de chaleur dû à la fois à la convection et à la conduction pure avec les mêmes conditions aux limites. Le rapport $N u$ s'exprime sous une forme adimensionnelle par :

$N u=V \theta-\frac{\partial \theta}{\partial Y}$.

De nombreux auteurs utilisent plus généralement un nombre de Nusselt moyen à travers un plan parallèle à l'absorbeur $(Y=$ constant $)$

$\overline{N u}(Y)=\frac{1}{l} \int_{0}^{l} N u \mathrm{~d} X$

Dans le cas de parois latérales $(X=0, X=l)$ isolées, $\overline{N u}(Y)$ est théoriquement indépendant de $Y$. On a alors :

$\overline{N u}(Y)=\overline{N u}(0)$.

Dans une résolution numérique cette égalité n'est vérifiée que de manière approchée, et l'écart $\overline{N u}(Y)-\overline{N u}(0)$ peut être utilisé pour tester la précision de la méthode. On notera enfin que $\overline{N u}(Y)=1$, dans le cas purement conductif où $V \equiv 0$.

Le calcul du transfert de chaleur a été effectué dans le cas $\operatorname{Pr}=0,7$ pour de nombreuses valeurs de $R a, \alpha$ et $l$. L'ensemble des résultats obtenus pour quelques valeurs de $R a$, telles que

$12500<R a<200000$,

sont portés en fonction de $\alpha$ sur les figures 7 à 12 pour des valeurs croissantes de $l(1<l<32)$. A $R a$ fixé chacune des représentations de $\overline{N u}$ en fonction de $\alpha$ présente un maximum pour $60^{\circ}<\alpha<80^{\circ}$, mais la position de ce maximum dépend de $R a$. La

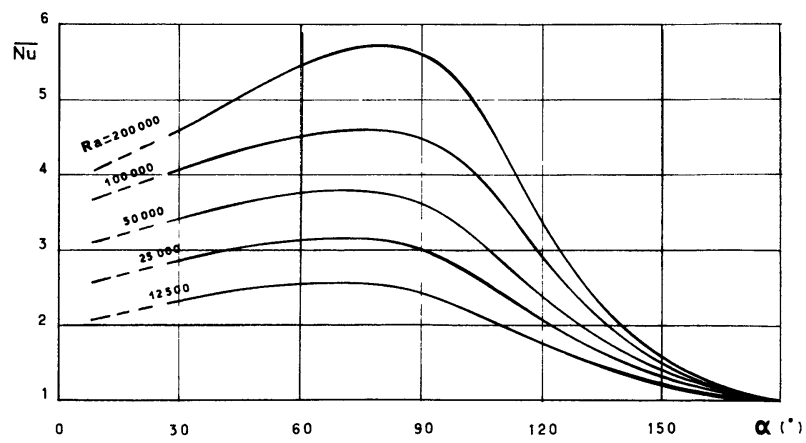

Fig. 7. - Evolution de $\overline{N u}$ en fonction de $\alpha(l=1)$.

[Evolution of $\overline{N u}$ in terms of $\alpha(l=1)$.]

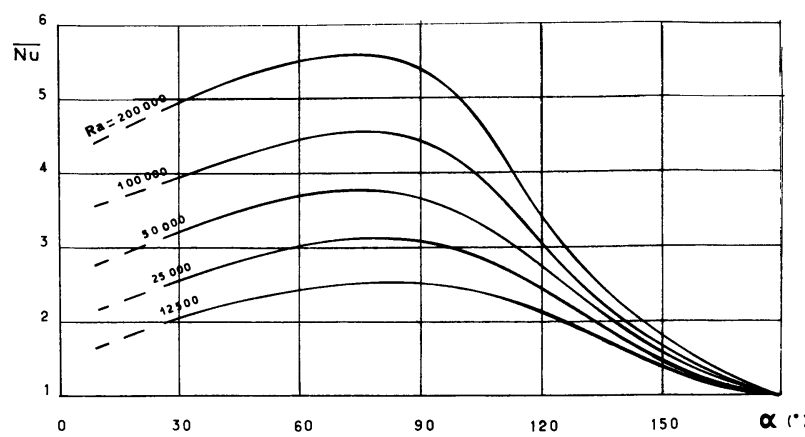

Fig. 8. - Evolution de $\overline{N u}$ en fonction de $\alpha(l=2)$.

[Evolution of $\overline{N u}$ in terms of $\alpha(l=2)$.]

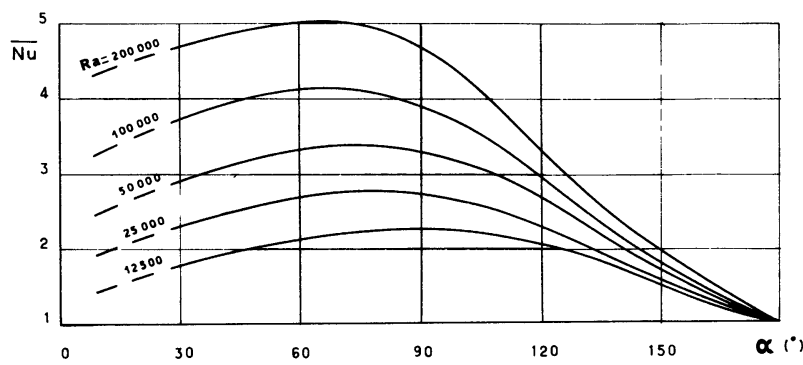

Fig. 9. - Evolution de $\overline{N u}$ en fonction de $\alpha(l=4)$.

[Evolution of $\overline{N u}$ in terms of $\alpha(l=4)$.]

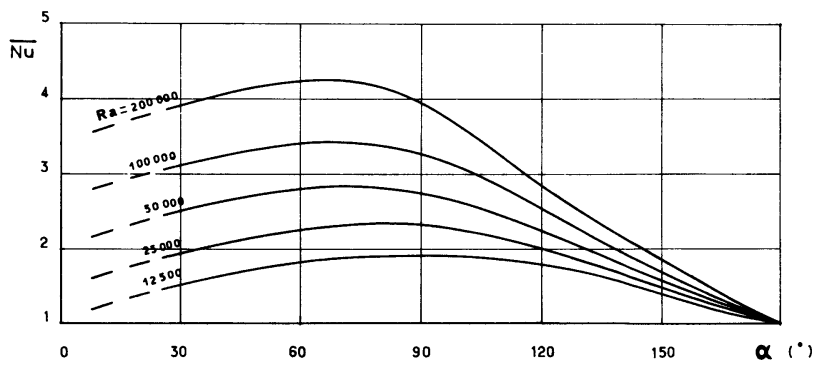

Fig. 10. - Evolution de $\overline{N u}$ en fonction de $\alpha(l=8)$. [Evolution of $\overline{N u}$ in terms of $\alpha(l=8)$.]

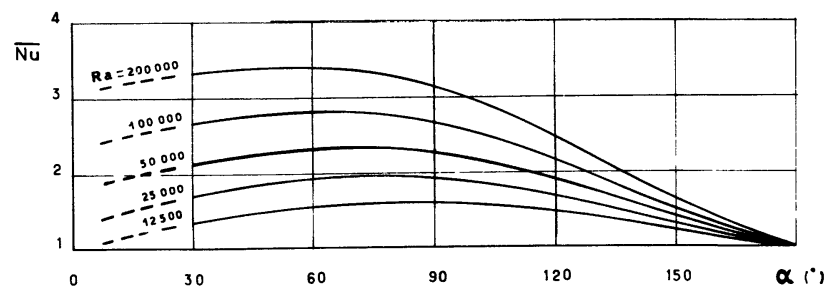

Fig. 11. - Evolution de $\overline{N u}$ en fonction de $\alpha(l=16)$.

[Evolution of $\overline{N u}$ in terms of $\alpha(l=16)$.]

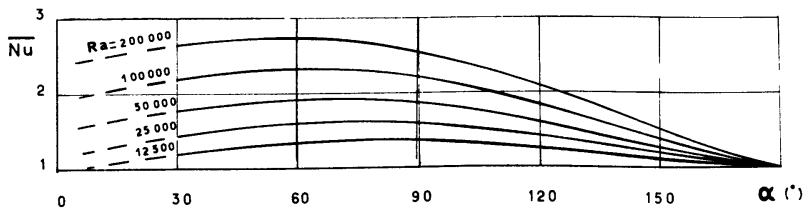

Fig. 12. - Evolution de $\overline{N u}$ en fonction de $\alpha(l=32)$.

[Evolution of $\overline{N u}$ in terms of $\alpha(l=32)$.] 
partie des courbes correspondant aux faibles valeurs de $\alpha$ est tiretée pour rappeler que le calcul n'est valable que pour $\alpha>\alpha_{c}$.

5.1 RECHERCHE DE CORRÉLATIONS DANS LE CAS VERTICAL. - De nombreuses corrélations ont été proposées dans la littérature, notamment dans le cas du régime de couche limite, pour les cavités verticales $\left(\alpha=90^{\circ}\right)$. Certaines, comme celles proposées par Eckert et Carlson [16], Dropkin et Somerscales [18] et par Seki et al. [19] ont été obtenues à partir d'études expérimentales. D'autres résultent des solutions numériques des équations de Navier-Stokes, comme celles de Mynett et Duxbury [20], Newell et Schmidt [21], Thomas et De Vahl Davis [17] et Yin et al. [22]. D'autres enfin, proposées par Raithby et al. [23] et par Emery et Chu [24], ont été obtenues à partir de solutions numériques des équations du mouvement utilisant l'approximation de couche limite.

Parmi ces corrélations, certaines $[16,21,22]$ obtenues dans le cas de l'air $(\operatorname{Pr} \backsim 0,7)$, ont été données sous la forme

$\overline{N u}=C G r^{m} l^{-n}$

proposée initialement par Jakob [25] en analysant les résultats expérimentaux de Mull et Reiher [26]. Cependant les résultats numériques obtenus en référence [15] pour différentes valeurs de $\operatorname{Pr}$ tendent à montrer qu'il est plus correct d'utiliser une corrélation basée sur $R a=G r P r$ plutôt que sur $G r$, comme en (5.1). Les résultats de la référence [15] nous amènent également à émettre quelques réserves sur l'interprétation des résultats expérimentaux proposée en [19], suivant laquelle $\overline{N u}$ serait proportionnel à $\operatorname{Pr}^{0,051}$ pour $4<\operatorname{Pr}<12500$.

On peut également douter de la validité de la corrélation proposée dans l'étude expérimentale donnée en [18] qui ne tient pas compte de l'influence du rapport de forme comme l'ont déjà signalé Raithby et al. [23]. D'une manière plus générale, les résultats des expériences dans lesquelles la variation de $R a$ est obtenue par une variation de la distance $H$ ne permettent pas d'isoler les influences de $R a$ et de $l$ et donc ne se prêtent pas aux corrélations faisant intervenir ces deux grandeurs.

Tableau I.

\begin{tabular}{lll}
\multicolumn{1}{c}{$A$} & \multicolumn{1}{c}{$a$} & \multicolumn{1}{c}{$b$} \\
- & - & - \\
0,23 & 0,269 & $-0,131$ \\
0,20 & 0,25 & $-0,111$ \\
0,13 & 0,3 & $-0,1$ \\
0,17 & 0,315 & $-0,265$ \\
0,216 & 0,263 & $-0,210$ \\
0,286 & 0,258 & $-0,238$ \\
0,29 & 0,25 & $-0,25$ \\
0,28 & 0,25 & $-0,25$ \\
0,29 & 0,25 & $-0,25$
\end{tabular}

En définitive, la forme des corrélations la plus réaliste nous semble être celle proposée en références $[17,19]$, soit

$\overline{N u}=A R a^{a} l^{-b}$.

En évaluant $A=C(P r)^{-m}$ dans les références [16, 21, 22 et 25], nous avons rassemblé dans le tableau I, les différentes valeurs des coefficients $A, a$ et $b$ de la relation (5.2), proposées dans la littérature par voie expérimentale, numérique ou théorique. On constate une dispersion très appréciable des valeurs de ces coefficients. On notera particulièrement la différence entre les valeurs de $b$ obtenues d'après les études expérimentales et qui sont voisines de 0,1 et celles prévues par les calculs qui se situent aux environs de 0,25 .

Comme on l'a signalé en référence [25] il est nécessaire d'avoir une grande précision sur $\mathrm{Nu}$ pour donner une évaluation correcte des coefficients $A$, $a$ et $b$. Une erreur de $2 \%$ sur $\overline{N u}$ peut entraîner un écart de l'ordre de $3 \%$ sur $a$ et $b$ et de l'ordre de $10 \%$ sur $A$. La corrélation proposée dans la présente étude s'écrit :

$\overline{N u}=0,29 R a^{0,25} l^{-0,25}$.

Cette expression est très voisine de celle obtenue par voie numérique en référence [17] ainsi que de celles résultant de la théorie de couche limite [23, 24].

5.2 RECHERCHE DE CORRÉLATIONS DANS LE CAS INCLINÉ. - Dans le cadre de la référence [1] une une recherche analogue de corrélations a été faite en représentant $\overline{N u}$ en fonction des paramètres $R a$ et $l$ pour différentes valeurs de l'inclinaison. Des corrélations sous la forme (5.2) n'existent qu'en dehors du régime conductif et pour $l \geqslant 4$. Les valeurs des coefficients $a$ et $b$ et de la constante $A$ obtenues alors pour $\alpha=30^{\circ}, 45^{\circ}, 60^{\circ}, 75^{\circ}, 90^{\circ}, 105^{\circ}$ et $120^{\circ}$, sont portées dans le tableau II. On remarquera que $b$ est pratiquement indépendant de $\alpha$. Par contre, $a$ diminue et $A$ augmente notablement lorsque $\alpha$ varie de $30^{\circ}$ à $120^{\circ}$.

Ce résultat montre un désaccord certain avec l'évolution proposée en référence [23] par la théorie

Auteurs
Exp. $\left\{\begin{array}{l}\text { Yin et al. }[22] \\ \text { Jakob }[25] \\ \text { Eckert et Carlson [16] }\end{array}\right.$
Num. $\left\{\begin{array}{l}\text { Newell et Schmidt }[21] \\ \text { Mynett et Duxbury [20] } \\ \text { Thomas et De Valh Davis [17] } \\ \text { Presents }\end{array}\right.$
Th. $\left\{\begin{array}{l}\text { Emery et Chu [24] } \\ \text { Raithby et Hollands [23] }\end{array}\right.$

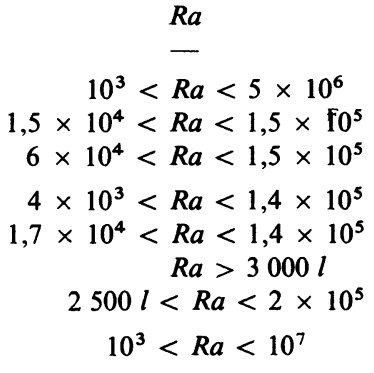

$R a$ $10^{3}<R a<5 \times 10^{6}$ $1,5 \times 10^{4}<R a<1,5 \times \mathrm{f0}^{5}$ $6 \times 10^{4}<R a<1,5 \times 10^{5}$ $4 \times 10^{3}<R a<1,4 \times 10^{5}$ $1,7 \times 10^{4}<R a<1,4 \times 10^{5}$ $R a>3000 l$ $2500 l<R a<2 \times 10^{5}$ $10^{3}<R a<10^{7}$ 
de couche limite, pour $70^{\circ}<\alpha<110^{\circ}$, où $A=0,29(\sin \alpha)^{1 / 4}$, et où $a=b=0,25$ quel que soit $\alpha$.

Remarque. - Il faut rappeler qu'étant donné le caractère bidimensionnel de la modélisation adoptée, les résultats présentés dans le tableau II ne sont valables que pour des configurations telles que $\alpha>\alpha_{c}$.

6. Conclusion. - On dispose d'une méthode de haute précision qui a été utilisée pour évaluer les transferts de chaleur convectifs dans une couche fluide inclinée, différentiellement chauffée, dans le cas d'un écoulement laminaire et bidimensionnel.

L'identification des frontières des différents régimes d'écoulement qui existent dans une telle couche a été réalisée.

On s'est intéressé particulièrement à des corrélations
Tableau II.

\begin{tabular}{|c|c|c|c|}
\hline$\alpha$ & $A$ & $a$ & $b$ \\
\hline - & - & - & - \\
\hline 30 & 0,117 & 0,33 & 0,25 \\
\hline 45 & 0,177 & 0,30 & 0,26 \\
\hline 60 & 0,262 & 0,27 & 0,27 \\
\hline 75 & 0,263 & 0,27 & 0,27 \\
\hline 90 & 0,29 & 0,25 & 0,25 \\
\hline 105 & 0,39 & 0,225 & 0,27 \\
\hline 120 & 0,538 & 0,18 & 0,25 \\
\hline
\end{tabular}

valables au-delà du régime conductif et pour les allongements $l \geqslant 4$.

Il existe alors des corrélations sous la forme $\overline{N u}=A R a^{a} l^{-b}$. Les valeurs des coefficients $A, a$ et $b$ ont été comparées à celles existant dans la littérature, notamment dans le cas $l=90^{\circ}$. L'influence de l'inclinaison sur ces coefficients a pu être déterminée, pour $\alpha_{c}<\alpha<120^{\circ}$.

\section{Bibliographie}

[1] Grondin, J. C., Contribution à l'étude de la Convection naturelle dans un capteur solaire plan. Doctorat de Spécialité, Université de Provence, Marseille (9 juin 1978).

[2] Hart, J. E., Stability of the flow in differentially heated inclined box, J. Fluid Mech. 47 (1971) 547-576.

[3] Korpela, S. A., A study on the effect of Prandtl number on the stability of the conduction regime of natural convection in an inclined slot, Int. J. Heat Mass Trans. 17 (1974) 215-222.

[4] Birikh, R. V., Gershuni, G. Z., Zhukhovitskit, E. M. and RudAKov, R. N., Hydrodynamic and thermal instability of a steady convective flow, P.M.M. 32 (1968) 256-263.

[5] UNNY, T. E., Thermal instability in differentially heated inclined fluid layers, J. Appl. Mech. Trans. ASME 39 (1972) 41-46.

[6] Chandrasekhar, S., Hydrodynamic and Hydromagnetic stability (Oxford University Press) 1961

[7] Ozoe, H., Sayama, H. and Churchill, S. W., Natural Convection in an inclined square channel, Int. J. Heat Mass Trans. 17 (1974) 401-406.

[8] Arnold, J. N., Catton, I. and Edwards, D. K., Experimental Investigation of Natural Convection in Inclined Rectangular Regions of differing Aspect Ratios, J. Heat Trans. 98C (1976) 67-71

[9] Bories, S. A. and Combarnous, M. A., Natural Convection in a sloping parous layer, J. Fluid Mech. 57 (1973) 63-79.

[10] Giblin, Transmission de la chaleur par convection naturelle, Collection de l'ANRT, Eyrolles, Paris (1974).

[11] MoRdChelles-Regnier, G. et Kaplan, G., Visualisation of natural convection on a plane wall and a vertical gap by differential interferometry. Transitional and turbulent regimes, Proceedings of the 1963 Heat Transfer and Fluid Mechanics Institute (Standford University Press).

[12] Gray, D. D. et Giorgini, A., The validity of the Boussinesq approximation for liquids and gases, Int. J. Heat Mass Trans. 19 (1976) 545-551.

[13] Ozoe, H., Yamamoto, K., Sayama, H. and Churchill, S. W., Natural Convection in an inclined rectangular channel heated on one side and cooled on the oposing side, Int J. Heat Mass Trans. 17 (1974) 1209-1217.

[14] Bontoux, P., Forestier, B. et Roux, B., Analyse et optima- lisation d'une méthode de haute précision pour la résolution des équations de Navier-Stokes instationnaires, J. Mécan. Appl. 2 (1978) 291-316.

[15] Roux, B., Grondin, J. C., Bontoux, P. and Gilly, B., On a high order accurate method for the numerical study of natural Convection in a vertical square cavity, Numerical Heat Transfer (à paraître).

[16] ECKert, E. R. G. and CARLSON, W. D., Natural convection in an air layer enclosed between two vertical plates with different temperatures, Int. Heat Mass Trans. 2 (1961) 106-120.

[17] Thomas, R. W. et De Vahl Davis, G., Natural convection in annular and rectangular cavities, A numerical study, Fourth Int. Congress in heat transfer, NC 2-4, Versailles (1970).

[18] Dropkin, D. and Somerscales, E., Heat transfer by natural convection in liquids confined by two parallel plates which are inclined at various angles with respect to the horizontal. J. Heat Trans. 87C (1965) 77-84.

[19] SEKi, N., FUKusako, S. and INABA, H., Visual observation of natural convective flow in a narrow vertical cavity, J. Fluid Mech. 84 (1978) 695-704.

[20] MynetT, T. A. A. et DuXbURY, D., Temperature distributions within enclosed plane air cells associated with heat transfer by natural convection, Fourth Int. Congress in heat transfer, NC 3-8, Versailles (1970).

[21] Newell, M. E. and Schmidt, F. W., Heat transfer by laminar natural Convection within rectangular enclosures, $J$. Heat Trans. 92C (1970) 159-168.

[22] Yin, S. H., Wung, T. Y. and CHEN, K., Natural Convection in an air layer enclosed within rectangular cavities. Int. J. Heat Mass Trans. 21 (1978) 307-315.

[23] Raithby, G. D., Hollands, K. G. T. and UNNY, T. E., Analysis of heat transfer by natural convection across vertical fluid layers, J. Heat Trans. 99C (1977) 287-293.

[24] Emery, A. and CHU, N. C., Heat transfer across vertical layers, J. Heat Trans. 87C (1965) 110-116.

[25] JАKOB, M., Free heat convection through enclosed plane gas layers, J. Heat Trans. 68 (1949) 189-193.

[26] Mull, M. and ReiHer, M., Gesund. Ing. (beihefte-reithe) 28 (1930) 1-28. 